\title{
La planeación didáctica basada en el contexto actual, eje fundamental para el desarrollo de competencias
}

\author{
Didactic planning based on the current context, a fundamental \\ axis for the development of competencies
}

Lorena Patricia Calics Salcedo

Universidad Metropolitana de Educación Ciencia y Tecnología. Ciudad de Panamá, Panamá

Recibido: 10/08/2021 Revisado: 22/09/2021 Aceptado: 26/09/2021 Publicado: 31/10/2021

\section{RESUMEN}

Este artículo tiene como objetivo mostrar la relevancia que representan las competencias en el contexto actual. La era de la sociedad del conocimiento (donde la globalización impuso nuevas exigencias) conlleva a que se le dé un giro a la educación y al aprendizaje de los educandos, en aras de responder de manera eficiente a los cambios que se dan constantemente en el mundo en el cual se vive inmerso. Toda esta situación obliga a transformar los sistemas educativos de una $u$ otra forma, es precisamente allí donde se vislumbra la necesidad de realizar una planeación didáctica contextualizada y basada en competencias, que permita la formación de individuos reflexivos, productivos, flexibles, creativos y competitivos, contribuyendo además a fortalecer en ellos la importancia del aprender a convivir juntos y a forjar valores que les ayude a desenvolverse de la mejor forma en el siglo XXI; alcanzando con esto su autorrealización, lo cual conlleve a que disfrute de una total emancipación. Palabras clave: Educación, aprendizaje, globalización, competencias.

\section{ABSTRACT}

This article is intended to show the relevance of competencies in the current context. The age of the knowledge society (where globalization imposed new demands) leads to a shift in education and learning for learners. in order to respond efficiently to the changes that are constantly taking place in the world in which one lives immersed. This whole situation obliges us to transform the educational systems in one way or another, it is precisely where the need for contextualized and competency-based didactic planning is envisioned, allowing the training of reflective, productive, flexible, creative and competitive individuals, It also contribute to strengthening in them the importance of learning to live together and to forging values that will help them to perform in the best way in the twenty-first century; thus achieving their self-realization, which leads to their enjoyment of total emancipation. Keywords: Education, learning, globalization, competencies. 


\section{INTRODUCCIÓN}

Continuamente el ser humano está enfrentando diversas situaciones que requieren de acciones pertinentes para generar cambios; los cuales se convierten en beneficios individuales o colectivos. Esto, lo hace debido a que el mismo contexto o sociedad en la que se desenvuelve se lo exige, y para ello ha tenido o tiene que poner en marcha un cúmulo de conocimientos y diversas habilidades, las cuales le van a facilitar su desempeño en el mismo.

Teniendo en cuenta este aspecto es cuando precisamente se reafirma el papel trascendental de la educación en la vida del hombre, la cual es entendida como el instrumento de cambio y desarrollo de toda nación, que proporciona a los ciudadanos su formación y transformación así mismos y a los medios sociales, naturales y culturales donde habita; permitiendo con ello el desarrollo continuo de las sociedades; partiendo de este antecedente, es cuando se visualiza la relevancia que cobra el sector educacional, y por ende el aprendizaje basado en competencias, el cual se fundamenta bajo las reglas de hoy, donde se hace necesario propender por una educación de calidad con fines sociales y en donde cada día los avances en los cuales se vive inmerso no dan tregua de quedarse rezagados, sino, que hay que estar a la vanguardia de ellos, ya que son indispensables para lograr dichos objetivos.

En base a los planteamientos anteriores, se puede resaltar que de hecho todos estos cambios a los cuales se ha visto sometido el ser humano, han sido la respuesta a la llamada globalización, la cual trajo consigo innumerables desafíos para la sociedad en general, entre ellos el hecho de comenzar a hablar sobre las competencias, cabe resaltar que este término no fue acuñado en principio por el sector educativo, este tuve su auge en el sector industrial, más específicamente alcanzo popularidad en los años 90, siglo XX, cuando se dio la revolución de las tecnologías de la información y las comunicaciones, de hecho con esto se le dio paso a la llamada sociedad del conocimiento, la era de la competitividad, lo cual condujo a aplicar nuevas pautas de desempeño en el sector empresarial y en el trabajo individual, trayendo consigo nuevos retos para ampliar el conocimiento y por ende permeando el proceso de enseñanza- aprendizaje, el cual se vio en la necesidad de estructurar un nuevo enfoque, basado en competencias.

El término competencia en uno de los más nombrados en la actualidad, este ha cobrado mucha relevancia en el sector educativo, donde se afirma que al educar basándose en un enfoque por competencias, se está trabajando en un proceso de aprendizaje para toda la vida. El Ministerio de Educaciòn Nacional MEN (s.f.) afirma "Las competencias son el conjunto de conocimientos, habilidades, actitudes que desarrollan las personas y que les permiten comprender, interactuar y transformar el mundo en el que viven" (p.10). y de hecho para que las personas puedan transformar ese mundo en el que viven deben enfrentarse a la nueva realidad, la cual ofrece un sin número de recursos, flujos de informaciones y experiencias tecnológicas sin precedentes, la educación deberá responder efectivamente, al gran cumulo de conocimientos y encontrar las estrategias para llevarlos a la práctica con un sentido ético, en aras de responder a los nuevos cánones impuestos en el siglo XXI, un mundo cada día en un constante caos, agitación y alta complejidad, donde la educación basada en competencias se convierte en la herramienta indispensable para enfrentarse de la mejor forma a estas nuevas tendencias. 
Teniendo en cuenta esto se puede afirmar:

En el enfoque por competencias, los conocimientos no son los más importantes, sino el uso que se hace de ellos en situaciones específicas de la vida personal, social y profesional. De esta manera las competencias requieren de una base sólida de conocimientos y teorías, pero las habilidades junto con las actitudes y valores serán las que darán movilidad o vida a las mismas (Morales, 2018, p.4).

Esto deja en evidencia que en una educación donde se propenda por potenciar las competencias, el proceso de enseñanza aprendizaje se debe resignificar, es por ello que el docente debe dejar de ser simplemente aquel que se enfocaba exclusivamente en lo cognitivo, redondeándolo solamente a un plano cuantitativo, ya que no daría resultado que al educando solo se le evalué el cumulo de conocimientos que tiene la capacidad de adquirir a lo largo de su vida. De hecho, el estudiante de la nueva era debe estar abierto al cambio, donde visualice lo indispensable de actualizar, elevar cada día más ese saber adquirido, y donde lleve a la praxis las habilidades y las actitudes arraigadas durante el proceso, lo cual le permita adaptarse de la mejor forma a un mundo en constante evolución.

Ese mundo del cual se habla en el nuevo milenio, necesita formar personas que no le tengan miedo a las nuevas tendencias, que sean capaces de aceptarlas y comenzar a hacer parte de ellas; en la era moderna nadie se puede quedar rezagado viviendo del pasado, hay que aprender a romper paradigmas. En cuanto a esto Harari (2018) sustenta que la educación cimento sus bases bajo una línea la cual no per- mitía ninguna reestructuración, pero en el siglo XXI, se abre la puerta para romper ese modelo impuesto, considerando que esta se encuentra en una época marcada por la revolución de las TIC, donde se hace inminente un cambio en el proceso educacional, el cual indudablemente va a estar marcado por constantes vicisitudes.

Teniendo en cuenta estos aspectos es que la educación de la nueva era debe reinventarse, es por ello que se ve en la necesidad de trabajar por establecer nuevos paradigmas, todo ello en aras de responder a la globalización y a las nuevas tecnologías, es precisamente allí donde recae la importancia de trabajar el proceso de enseñanza aprendizaje con un enfoque por competencias, lo cual coadyuve a alcanzar mejores resultados y de esta manera estar a la par con las demandas de la competitividad que plantean las nuevas políticas. De hecho, para que la educación pueda responder de manera eficaz a todos estos nuevos desafíos se plantea que esta debe cimentarse sobre cuatro aprendizajes que le ayudaran a cada ser humano a adquirir los conocimientos, habilidades y actitudes, necesarias para desempeñarse en el contexto. Delors (1996) plantea:

Se habla de aprender a conocer, es decir, adquirir los instrumentos de la comprensión; aprender a hacer, para poder influir sobre el propio entorno; aprender a vivir juntos, para participar y cooperar con los demás en todas las actividades humanas; por último, aprender a ser, un proceso fundamental que recoge elementos de los tres anteriores. Por supuesto, estas cuatro vías del saber convergen en una sola, ya que hay entre ellas múltiples puntos de contacto, coincidencia e intercambio (pp. 95-96). 
En el entorno educativo actual aún se sigue vivenciando la prevalencia en las aulas educativas por el aprender a conocer, donde el aprender a hacer lo llevan a la praxis en menor proporción, y los otros dos pilares planteados aún los siguen dejando de lado totalmente. En el aprendizaje por competencias esta situación se convierte en un grave error, dado que en un sistema educativo donde se propenda por formar seres competentes estos cuatro pilares deben ser abordados en la misma medida, en aras de que el sujeto en formación logre aprehenderse de un conocimiento tanto teórico como práctico, además de poseer las habilidades cognitivas, procedimentales y actitudinales, que le permitan alcanzar una formación integral, lo cual contribuya a que este se desenvuelva de una manera competente en el contexto en el cual vive inmerso, respondiendo de esta forma a las exigencias del contexto contemporáneo.

Ahora bien, para la formación de ese ser competente sea hace inminente acabar definitivamente con el aprendizaje memorístico, donde al educando recibe solo un cumulo de conocimientos el cual no logra llevar a la práctica y donde no se preocupa por potenciar la convivencia pacífica, el trabajo en equipo, la libertad, la democracia, además no asimila la importancia de la solidaridad, respeto por los demás, etc, convirtiéndose este tipo de enseñanza en algo inerte, que no le permite salir de lo rutinario, y por ende no responde a sus intereses ni a sus expectativas, lo cual dificulta que el discente potencie sus características tanto sociales como individuales. Es precisamente atendiendo a esto que resulta como pieza clave para la formación de seres competentes trabajar en cada aula educativa los cuatro pilares planteados, y no olvidar que en este tipo de aprendizaje todo debe girar en torno al estudiante, él es el principal protagonista, y la función del docente radica en ser un guía, cuyo principal objetivo se debe centrar en pro de la formación de seres emancipados, empoderados de las dificultades que atraviesa el mundo y dispuestos a aportar todo su conocimiento, con un sentido social y ético en aras de forjar una mejor sociedad.

Ahora bien, para la formación de ese ser competente sea hace inminente acabar definitivamente con el aprendizaje memorístico, donde al educando recibe solo un cumulo de conocimientos el cual no logra llevar a la práctica y donde no se preocupa por potenciar la convivencia pacífica, el trabajo en equipo, la libertad, la democracia, además no asimila la importancia de la solidaridad, respeto por los demás, etc, convirtiéndose este tipo de enseñanza en algo inerte, que no le permite salir de lo rutinario, y por ende no responde a sus intereses ni a sus expectativas, lo cual dificulta que el discente potencie sus características tanto sociales como individuales. Es precisamente atendiendo a esto que resulta como pieza clave para la formación de seres competentes trabajar en cada aula educativa los cuatro pilares planteados, y no olvidar que en este tipo de aprendizaje todo debe girar en torno al estudiante, él es el principal protagonista, y la función del docente radica en ser un guía, cuyo principal objetivo se debe centrar en pro de la formación de seres emancipados, empoderados de las dificultades que atraviesa el mundo y dispuestos a aportar todo su conocimiento, con un sentido social y ético en aras de forjar una mejor sociedad.

Para lograr lo expuesto en el apartado anterior, se hace urgente darle un nuevo giro a la planeación didáctica, con miras de responder a las exigencias del 
La planeación didáctica basada en el contexto actual, eje fundamental para el desarrollo de competencias

nuevo milenio. Ascencio (2016) afirma:

La planeación didáctica implica la organización de un conjunto de ideas y actividades que permiten desarrollar un proceso educativo con sentido, significado y continuidad. Constituye un modelo o patrón que permite enfrentar de forma ordenada y congruente, situaciones a las que el estudiante se enfrentará en su vida profesional o cotidiana y en el caso del profesor, a su práctica docente (p.109).

Enfocándose en este planteamiento, se acentúa mucho más la necesidad de resignificar el aspecto en mención, con el firme propósito de potenciar el tema de las competencias, $\mathrm{y}$ es que este cambio resulta indispensable, más en la nueva realidad a la cual se ve sometida la humanidad, debido a la terrible pandemia que aqueja al mundo, precisamente ese caos, incertidumbre que ha conllevado toda esta situación a nivel global, exige una reestructuración, es allí donde entra y cobra importancia planear acorde a la nueva realidad ( la educación virtual), ya que ella se ha convertido en la herramienta con la cual cuentan las sociedades para tratar de seguir con un ciclo educativo normal. Salvador (2017) manifiesta:

La escuela como centro de cultura debe de germinar y generar las posibilidades para hacer frente a los grandes cambios que exige el proceso formativo integral del ser humano. En los diferentes niveles educativos debe de existir el compromiso impostergable de reconocer y reencauzar la dirección del proceso de enseñanza - aprendizaje desde las nuevas exigencias de nuestra realidad social. Es decir, brindar a los estudiantes los elementos suficientes y
Calics, L.

necesarios para que ellos sean los protagonistas del cambio y transformación de su contexto inmediato (p.88).

Teniendo como base lo señalado en el apartado anterior, se hace evidente la urgencia de una restructuración en la planeación didáctica, en fin de responder a la educación virtual, donde cabe añadir que primero se debe comenzar en acabar con los currículos que se centran solamente en asignaturas, donde predomina el aprendizaje memorístico, de hecho, el desafío de la realidad actual, es construir currículos donde el eje o transversalidad de este se centre en las dimensiones del ser humano, esto conllevaría a enfocarse en potenciar proyectos de aula, los cuales pasarían a convertirse en un aspecto fundamental en la educación en mención, y es que la idea es diseñar ambientes pedagógicos donde se tenga en cuenta el contexto, las particularidades e intereses de los educandos, se les permita la participación activa, se planteen experiencias de aprendizaje significativas, con tareas auténticas, las cuales se conviertan en un reto o desafío para los aprendices y que estos a su vez lo puedan evidenciar en la puesta en práctica del saber adquirido, coadyuvando con ello a forjar un ser autónomo, colaborativo, crítico, reflexivo, resiliente, flexible e innovador, capacitado para asumir los retos del entorno desde una perspectiva holística y transdiciplinar. Es inminente que en esta nueva estructuración curricular el educando debe pasar a convertirse en el protagonista de su propio proceso de aprendizaje, fortaleciendo con ello su competencia de aprender a aprender de manera constante, otras situaciones que se deben tener en cuenta es el nuevo rol que debe desempeñar el docente, el cual pasa de ser el transmisor de conocimientos a ser un acompañante, el mediador, el gestionador de los ambientes de aprendizaje. 
Es por ello que se vuelve a afirmar la necesidad de enfatizar en realización de esta clase de planeaciones, la cual demanda inminentes reestructuraciones curriculares, contribuyendo a fortalecer el desarrollo de competencias, es en ese punto cuando se proponen algunas estrategias que conlleven a mejorar este aspecto, algunas serían, involucrar a la comunidad educativa, motivándolos a que piensen, reflexionen acerca de los cambios o transformaciones necesarios para afrontar la nueva realidad, realizar un diagnóstico de manera interna, de las carencias, necesidades y expectativas del currículo, tener muy en cuenta los lineamientos macro, meso y micro que brinden un norte para realizar esta nueva reestructuración, determinar los resultados de aprendizaje que ayuden a establecer el perfil del egresado, tener presente el plan de estudios, el cual debe ir en todo momento afín con los elementos de la competencia y con los resultados de aprendizaje, además de tener claridad en el programa del área o la asignatura y cómo van a ser las actividades de enseñanza- aprendizaje, plantear lineamientos para la evaluación de los aprendizajes, lo cual deje entrever el resultado del aprendizaje, asimismo organizar un sistema de seguimiento, que permita reafirmar que el aprendizaje por competencias debe ser versátil, flexible, pero sobre todo adaptado al contexto.

Se añade que la evaluación también debe dar un giro, acabando con la imagen o concepción de coercitiva, de aprobatoria, redefiniéndose como parte fundamental de este proceso, pero desde una concepción formativa, la cual permita dejar al descubierto las falencias y de esta manera se pueda trabajar en ellas, alcanzando de esta forma un mejor desempeño o resultado de aprendizaje. En base a ello se hace notable la importancia que cobra las nuevas reestructuraciones en el proceso educativo, resaltando el papel preponderante que juega en este caso las planeaciones didácticas. Salvador (2017) plantea:

El plan de clase recobra notable importancia al convertirse en el eje transversal que garantiza al docente aterrizar las actividades de manera sistémica y sistemática. En tal razón, es una tarea permanente de los educadores indagar, innovar y transformar su práctica educativa a partir de los paradigmas contemporáneos que favorecen la formación integral de la personalidad de los estudiantes (p.88).

Partiendo de este postulado, se proponen algunos de los aspectos que más se deberían tener en cuenta para la planeación didáctica en el contexto educativo virtual, en aras de potenciar competencias acordes a las demandadas del siglo XXI, ¿Quién aprende?, ¿cuál es la nueva realidad en estos tiempos?, ¿en qué sociedad se está involucrando este ser que aprende?, ¿cuál es el aporte que deben hacer las diferentes disciplinas?, ¿cuál es la importancia de la autorrealización del estudiante?, teniendo en cuenta todo esto se debe trabajar en busca de realizar una planeación didáctica flexible, enfocada en preparar para enfrentarse al caos, a la incertidumbre, a la adversidad, que forme a un discente autónomo, capaz de pensar por sí mismo, y que sea resiliente. Rodriguez (2012). manifiesta:

La planeaciòn didàctica es de suma importancia en el quehacer del profesor, porque aquì es donde el profesor refleja su creatividadal al momento de seleccionar y organizar actitivades de aprendizaje con enfoques que permitan al estudiante desarrollar competencias y actitudes 
crìticas sobre lo que aprende (p.20).

Ahora bien, los docentes en esta nueva realidad a la que se le ha llamado la educación virtual, deben enfatizar en ciertos aspectos en aras de potenciar el desarrollo de competencias, mediante la planeación didáctica, es por ello que deben iniciar reconociendo y planteando el propósito de aprendizaje, ósea lo que se quiere lograr, para ello hay que tener en cuenta las competencias, las capacidades de aprendizaje, el desempeño y la evidencias, para así poder elaborar los criterios de evaluación, seguidamente proponer una experiencia de aprendizaje, donde se tenga en cuenta un diagnóstico, que debe ser realizado con anterioridad, cuyo fin se centra en recoger información acerca de qué saben los educandos, las experiencias que tienen con respecto a la temática a trabajar, cuáles son sus mayores intereses, etc, además no dejar de lado los materiales con los que se cuentan, pero sobre todo con los que cuenta el educando, ya que siempre este deberá estar en el primer lugar, se anota que se hace necesario mirar el currículo, para enfocarse en él de acuerdo al área, teniendo claro que es indispensable a la hora de planificar. Al centrarse en este nuevo modelo para la planificación didáctica cabe señalar algunos de las estrategias que más están siendo utilizadas, y que han logrado impactar en el mejoramiento de los resultados, entre ellas se encuentra el aprendizaje basado en problemas, el aprendizaje colaborativo, el aprendizaje basado en proyectos, el aprendizaje basado en estudio de casos, etc, todo esto enfocado en el contexto en el cual vive inmerso el educando. De hecho las nuevas reestructuraciones a las que se ha visto abocada la planeación didáctica para el desarrollo de competencias en la era de la educación virtual, han sido motivo de mucha polémica, porque aún se sigue sumergido en el aprendizaje tradicional, es muy difícil para la sociedad romper paradigmas, donde muchos de los miembros de las comunidades educativas siguen atónitos ante todos estos cambios, dado que el mundo no estaba preparado para ello, y de un momento a otro les ha tocado de una u otra manera enfrentarse a esta nueva realidad, la cual no terminan de asimilar.

Todo esto demuestra la necesidad de formar personas que se desenvuelvan de una manera competitiva en un mundo cambiante, lo cual exige hablar de competencias, a consecuencia de esto las políticas educativas hacen evidente la urgencia de establecer modelos curriculares que tengan como eje principal este enfoque. Por eso se hace inminente que todas las áreas del saber abordadas desde el mismo currículo institucional y plasmadas en la planeación didáctica apunten a entregarle a la sociedad seres competentes. De hecho, se parte de lo evidente, donde se comprende que el proceso educativo no se quedó estático y no esta excepto de estos cambios, es por ello que se abre la necesidad de buscar las alternativas para formar a toda la comunidad educativa (docentes, padres de familia, estudiantes) en las nuevas tendencias, entre ellas las plataformas tecnológicas, las cuales representan un medio eficaz para avanzar sin necesidad de salir de casa. Al respecto Semana (2020) sustenta que es inminente asimilar el cambio que ha sufrido la educación, por lo cual se hace urgente redireccionarla. Es allí donde se debe resignificar el nuevo papel que debe afrontar el padre de familia, el estudiante y por ende la escuela. Además, no se puede desconocer que la interacción y la comunicación entre los integrantes de este sistema se transformó, reconociendo con ello que ahora se encuentran sujetos al uso de plataformas tecnológicas lo cual coadyuva a darle continuidad al proceso educativo, y a la vez deja como resultado distintas formas de actuar y pensar, pero 
que al final permiten el acompañamiento y constante apoyo desde casa, logrando de esta forma estar acorde a las nuevas exigencias.

Por esta razón, los medios que se utilizan para la interacción en el aula constituyen un puente entre la cultura del contexto, los claustros educativos y la que se genera a través del trabajo, la cual siempre debe enfocarse en que los discentes cimienten el conocimiento, de tal manera que se convierta en la herramienta principal para resolver no solo las actividades planteadas dentro del aula, sino, que también les sirva para afrontar de la mejor forma las situaciones que van a vivenciar fuera de ella, claro está con unos conocimientos, actitudes y valores que conlleven a que enfrenten con éxito su propia realidad.

Pero es aquí, en este punto donde hay que hacer un alto en el camino y determinar la importancia que cobran algunas de las nuevas tendencias en el sector educacional, entre ellas la necesidad de articular las TIC en todos los niveles y comunidades educativas, de hecho al analizar minuciosamente este aspecto, es cuando se comienza a notar el abismo de desigualdades existentes entre muchos sectores, por ejemplo, entre el rural y el urbano, dejando en evidencia las desventajas a las cuales se ven expuestos muchos de los educandos en este nuevo modelo de enseñanza- aprendizaje, impuesto precisamente por las situaciones adversas que está atravesando la sociedad(pandemia actual.

Se puede concluir diciendo que la educación ha representado y va a representar en todos los tiempos el medio más poderoso que tiene un ser humano para formarse e integrase de una manera productiva a la sociedad en la que se vive inmerso actualmente. De allí la importancia de recibirla equitativa y de calidad, la cual conlleve a el desempeño eficiente de los seres humanos en ambientes complejos, es por ello que resulta relevante el modelo educativo basado en competencias, el cual pretende la formación de personas que puedan desempeñarse de la mejor forma en un mundo en constante dinamismo y evolución.

La educación del siglo XXI se debe centrar en el desarrollo de las competencias, un enfoque que enfatiza en la flexibilización y la autorrealización, en aras de forjar un ser que logre una total emancipación.

Los sistemas educativos han sufrido con el paso de los años ciertas transformaciones, los cuales han logrado que la educación se vivencie de una forma diferente. El mundo moderno trajo consigo nuevos requerimientos para el campo educativo, es por ello que se hace necesario romper ciertos paradigmas y buscar mediante las TIC nuevas formas de enseñanza- aprendizaje.

Se da paso a la era digital y con ello a un sin número de herramientas que le permiten al educando adentrarse en otra forma de aprendizaje, las nuevas tendencias educativas juegan un papel de suma relevancia en el estudiante de hoy en día, ya que estas representan el puente para mantenerse en el nivel que demanda la sociedad actual.

Para potenciar el ser que demanda la era moderna se urge redireccionar todo el proceso educativo, y más cuando se hace imperante atender a las nuevas exigencias del contexto, como lo es el confinamiento, distanciamiento social, cierre de escuelas. De hecho, para que ese cambio emerja, resulta imprescindible tener muy en cuenta las nuevas tendencias y necesidades, es precisamente allí donde se hace urgente darle un nuevo giro 
al diseño curricular y por ende a las prácticas áulicas, con el firme propósito de educar respondiendo al mundo contemporáneo.

La reestructuración curricular pasa a convertirse en el factor esencial para afrontar la nueva realidad en la que se encuentra sumergida la sociedad debido a la pandemia actual (covid 19).

La planeación didáctica en su nueva reestructuración se debe caracterizar por ser flexible, donde se trabaje por diseñar estrategias que conlleven a crear situaciones significativas, vivenciales y contextuales, las cuales respondan a los intereses y necesidades de los educandos, lo cual permita trazar propósitos de aprendizaje donde se implementen actividades auténticas que conlleven a realizar una evaluación procesual y formativa coadyuvando a desarrollar el pensamiento sistémico y crítico.
La escuela, los estudiantes, los padres de familia y los docentes cobran un nuevo rol, cada uno adquiere un alto grado de responsabilidad, evidenciándose a través de la autonomía que debe adquirir el discente, el sentido de compromiso del padre yel papel de orientador $\mathrm{u}$ acompañante en el proceso del maestro.

Las TIC se convierten en la herramienta principal que media el proceso educativo a la hora de impartir las clases, enviar las evidencias del trabajo propuesto y las retroalimentaciones dadas por los docentes.

La brecha existente en cuanto al tema digital sigue predominando en muchos sectores, lo que deja entrever la desigualdad existente más que todo en los grupos donde prevalece un alto índice de pobreza y vulnerabilidad.

\section{REFERENCIAS BIBLIOGRÁFICAS}

Ascencio, C. (2016). Adecuación de la Planeación Didáctica como Herramienta Docente en un Modelo Universitario Orientado al Aprendizaje. Revista Iberoamericana sobre Calidad, eficacia y Cambio en Educaciòn, 14(3), 109-130.

https://www.redalyc.org/pdf/551/55146042006.pdf

Delors, J. (1996). La educaciòn encierra un tesoro. España: Santillana. http://innovacioneducativa.uaem.mx:8080/innovacioneducativa/web/Documentos/educacion_tesoro.pdf

Harari, Y. (2018). Los robots obligaràn a las personas a reinventarse cada diez años. Barcelona, España: Universidad Internacional de Valencia. https://www.elperiodico.com/es/ocio-y-cultura/20180830/entrevista-yuval-noah-harari-21-lecciones-siglo-xxi-6963573

Ministerio de Educación Nacional (MEN). (s.f.). Revoluciòn Educativa, programa para el desarrollo de competencias.https://www.mineducacion.gov.co/1759/articles-217596_archivo_pdf_desarrollocompetencias.pdf 
Morales, R. (2018). La planeacion de la enseñanza-aprendizaje, competencia que fortalece el perfil docente. Revista Iberoamericana para la investigaciòn y el Desarrollo Educatico, 8(16), 1-24. http://www.scielo.org.mx/pdf/ride/v8n16/2007-7467-ride-8-16-00311.pdf

Rodrìguez, C. (2012). La Planeaciòn como Recurso Didàtico en el Proceso de Enseñanza en la Escuela Primaria. (Tesis de pregrado). http://200.23.113.51/pdf/28698.pdf

Salvador, J. (2017). La planeaciòn de clase; una tarea fundamental en el trabajo docente. Maestro y Sociedad, 14(1), 87-96.

https://educrea.cl/wp-content/uploads/2018/10/DOC1-planeacion-tarea-fundamental.pdf

Semana. (2020). Los retos que plantea el coronavirus para la educaciòn en Colombia. Bogotà, Colombia: Semana.

https://www.semana.com/nacion/articulo/los-retos-que-plantea-el-coronavirus-para-la-educacion-en-colombia/659653/ 